\section{rev Psi}

Revista de Psicología (UNLP)

https://revistas.unlp.edu.ar/revpsi

\title{
Usos de objetos y anticipaciones corporales en el desarrollo temprano
}

\author{
Maximiliano Vietri ${ }^{1} \quad$ Nicolás Alessandroni ${ }^{1,2} \quad$ María Cristina Piro $^{1}$
}

\section{Correspondencia}

mvietri@psico.unlp.edu.ar

Filiaciones institucionales

${ }^{1}$ Facultad de Psicología, Universidad Nacional de $\quad{ }^{2}$ Facultad de Psicología, Universidad Autónoma La Plata (UNLP, Argentina)

de Madrid (UAM, España)

\section{Resumen}

Existe evidencia empírica de que las interacciones diádicas contribuyen con la emergencia de la comprensión intencional. No obstante, poco se sabe acerca del rol que las interacciones triádicas (adulto-objeto-bebé) tienen en este proceso. Presentamos un análisis cualitativo de cuatro observaciones realizadas en el hogar, en Ensenada (Buenos Aires, Argentina). Dichas observaciones fueron un recorte de un estudio más amplio en el que se observaron, una vez por mes a lo largo de 7 meses, las interacciones de bebés con sus tutores y objetos. Los resultados muestran que los bebés anticipan corporalmente las acciones que los adultos realizan empleando objetos en situaciones de interacción triádica (adulto-objeto-bebé). La participación de los bebés en interacciones triádicas contribuye con la emergencia de anticipaciones corporales cada vez más complejas, coordinadas con el comportamiento de los demás y ajustadas a los usos canónicos de los objetos. Se defiende que estas anticipaciones son casos de comprensión intencional enactiva.

\section{Palabras clave}

desarrollo de la comprensión intencional | anticipaciones corporales | interacciones triádicas | usos de objetos

\section{Cómo citar}

Vietri, M., Alessandroni, N. y Piro, M.C. (2021). Usos de objetos y anticipaciones corporales en el desarrollo temprano. Revista de Psicología. HTTPS://DX.DOI. ORG/10.24215/2422572XE089

\section{Proceso editorial}

Recibido

30 sep. 2020

Aceptado

6 abr. 2021

Editor

Ana Moreno-Núñez | Facultad de Psicología, Universidad Autónoma de Madrid (España)
ISSN

2422-572X

Licencia

Licencia de Cultura Libre CC-BY 4.0

(Compartir - Adaptar - Atribuir)

RevPsi es una publicación de la

Facultad de Psicología (Universidad

Nacional de La Plata, Argentina) 


\section{Usos de objetos e antecipações corporais no desenvolvimento na primeira infância}

\section{Resumo}

Há evidências empíricas de que as interações diádicas contribuem para o surgimento de um entendimento intencional. Entretanto, pouco se sabe sobre o papel que as interações triádicas (adulto-objeto-bebê) desempenham neste processo. Apresentamos uma análise qualitativa de quatro observações feitas em casa, em Ensenada (Buenos Aires, Argentina). Estas observações fizeram parte de um estudo mais amplo no qual as interações dos bebês com seus cuidadores e objetos foram observadas uma vez por mês durante 7 meses. Os resultados mostram que os bebês antecipam fisicamente as ações que os adultos realizam utilizando objetos em situações de interação triádica (adultoobjeto-infantil). A participação dos bebês nas interações triádicas contribui para o surgimento de antecipações corporais cada vez mais complexas, coordenadas com o comportamento dos outros e ajustadas aos usos canônicos dos objetos. Argumenta-se que estas antecipações são casos de compreensão intencional inativa.

\section{Palavras-chave}

desenvolvimento da comprensão intencional | anticipações corporais | interações triadicas | uso de objetos

\section{Object uses and bodily anticipations in early development}

\section{Abstract}

There is empirical evidence that dyadic interactions contribute to the emergence of intentional understanding. However, little is known about the role that triadic (adultobject-baby) interactions play in this process. We present a qualitative analysis of four observations made at home in Ensenada (Buenos Aires, Argentina). These observations were part of a larger study in which infants' interactions with their caregivers and objects were observed once a month for 7 months. The results show that infants anticipate with their bodies the actions that adults perform using objects within triadic interactions (adult-object-infant). Infants' participation in triadic interactions contributes to the emergence of increasingly complex bodily anticipations that are coordinated with the behaviour of others and adjusted to the canonical uses of objects. It is argued that these anticipations are cases of enactive intentional understanding.

\section{Keywords}

development of intentional understanding | bodily anticipations | triadic interactions | use of objects 


\section{Aspectos destacados del trabajo}

- Los bebés anticipan corporalmente las acciones que los adultos realizan con objetos.

- Las anticipaciones son casos de comprensión intencional enactiva que tienen lugar en interacciones triádicas.

- Los bebés realizan anticipaciones corporales con distinto grado de complejidad.

- La intersección entre desarrollo de la comprensión intencional e interacciones triádicas merece ser explorada en futuros trabajos.

La comprensión intencional involucra la capacidad de entender, describir y/o predecir el comportamiento propio y ajeno (Spaulding, 2018). En psicología y filosofía esta capacidad fue tradicionalmente explicada desde el cognitivismo clásico. Desde dicho paradigma, la cognición (incluida la cognición social) involucra el procesamiento de información proveniente de un mundo predeterminado en forma de computaciones simbólicas (i.e., representaciones mentales). Por ejemplo, desde la teoría de la teoría de la mente (Gopnik y Wellman 1992/2002), se sostiene que nuestra capacidad para comprender a los demás se basa en una teoría que poseemos acerca de la mente y su relación con el mundo. Esta teoría nos proveería de un conocimiento general, de tipo legaliforme, a partir del cual generaríamos deducciones para explicar y predecir los distintos comportamientos. La técnica que se constituyó como paradigma de indagación de la teoría de la mente en niños/as fue el Test de Falsa Creencia (Wimmer y Perner, 1983). Si bien esta tarea ha tenido distintos formatos, básicamente consiste en un problema que se plantea a modo de una historia para evaluar si los/as niños/ as de al menos 13 (Surian et al., 2007), 15 (Onishi y Baillargeon, 2005) o 24 meses (Gopnik y Wellman, 1992/2002) logran atribuir una falsa creencia a un personaje en relación a un estado de hechos que ha cambiado en su ausencia. Es decir, se trata de un instrumento que permite indagar si los niños/as pueden representar los estados mentales de otra persona desde el punto de vista de un observador externo.

Posiciones más novedosas coinciden en redefinir la comprensión intencional en términos de un proceso interactivo, corporeizado y práctico (Abramova y Slors, 2015; Alessandroni, 2020, en prensa; De Bruin y Kästner, 2012; De Bruin et al., 2011; Hutto, 2011; Lindblom, 2020; Reddy, 2008). Por ejemplo, Abramova y Slors (2015) proponen que la comprensión intencional debería ser entendida como un caso de coordinación de los comportamientos. Desde este punto de vista, comprender las intenciones de otra persona es equivalente a actuar coherentemente con sus acciones en un contexto determinado y no a la representación "en la cabeza" de sus estados mentales (Alessandroni, 2020). Quienes apoyan este corrimiento conceptual 
rechazan la metodología individualista, excesivamente experimental (i.e., carente de validez externa) y socialmente desapegada de las perspectivas estándar (De Jaegher et al., 2010; Reddy, 2008; Reddy y Morris, 2004; Schilbach et al., 2013). En su lugar, sugieren investigar el desarrollo de la comprensión intencional observando interacciones sociales en escenarios ecológicos (e.g., hogar o jardín de infantes) y considerando las prácticas socio-materiales donde la comprensión intencional se desarrolla y opera (Alessandroni, 2020). En otras palabras, se busca atender al aspecto práctico de la cognición social, es decir, qué es lo que hacen los sujetos cuando comprenden intencionalmente a los demás.

El giro conceptual y metodológico que tiene lugar dentro del terreno de debates sobre la comprensión intencional permite explicarla emergencia de dicha capacidad desde los primeros meses de vida y abarcar fenómenos interactivos que antes eran desatendidos (e.g., comportamientos engañosos y humorísticos) (Mireault y Reddy, 2015; Newton et al., 2000; Reddy, 1991, 2001; Reddy y Mireault, 2015). En esa línea, Reddy et al. (2013) documentaron un comportamiento que indica que los bebés comprenden a los demás de forma corporeizada y no inferencial desde sus dos meses de vida: los ajustes corporales anticipatorios (véase también, Fantasía et al., 2016). En su investigación, Reddy et al. (2013) reportaron que los bebés anticipan el comportamiento de los adultos mediante ajustes corporales (e.g., levantando los brazos antes de ser alzados). La observación de este tipo de comportamiento ha llevado a diversos autores a postular que la comprensión intencional se desarrolla inicialmente en interacciones diádicas (adulto-bebé) (e.g., Hobson, 2002; Reddy, 2008, para una revisión sistemática de estos aportes, véase Vietri et al., 2019).

Otros estudios de la psicología del desarrollo argumentan a favor de la existencia de interacciones triádicas tempranas, donde los adultos introducen a los bebés en nichos de acción conjunta (Rodríguez et al., 2018; Rossmanith y Reddy, 2016). En este tipo de interacciones se generan regularidades interactivas vinculadas con el uso funcional de los objetos que contribuyen con la comprensión mutua y la coordinación de las conductas del adulto y del bebé (Rossmanith et al., 2014). Todo ello sugiere que indagar el rol de la materialidad es relevante para comprender el desarrollo temprano de la comprensión intencional (Alessandroni, 2020; Costall, 2013; Striano y Reid, 2006).

Por ejemplo, distintos estudios trasversales de seguimiento de la mirada mostraron que los bebés anticipan visualmente el accionar de los adultos (i.e., miran la meta hacia la que se dirige el objeto antes de que este arribe allí) desde los 6 meses de edad (Hunnius y Bekkering, 2010; Kochukhova y Gredebäck, 2010). En esos estudios de laboratorio, los bebés eran expuestos a distintos videos en los que un actor adulto empleaba un objeto (e.g., usaba una taza para beber) e interesantemente lograron anticipar visualmente hacia dónde se dirigía la acción del adulto cuando este último usaba el objeto canónicamente (i.e., miraban los labios del adulto antes 
de que este se llevara la taza a la boca) (Hunnius y Bekkering, 2010) y cuando la situación era típica (i.e., los bebés no exhibieron anticipaciones cuando los objetos eran autopropulsados artificialmente) (Kochukhova y Gredebäck, 2010). En otro estudio (Green et al., 2016) se analizaron las respuestas visuales de bebés chinos y suecos de 8 meses de edad cuando un actor adulto comía usando una cuchara y usando palillos chinos. Los autores hallaron que los bebés chinos anticiparon el uso de ambos objetos (puesto que la cuchara no les resultaba totalmente ajena), aunque exhibieron mayores tiempos de anticipación cuando el actor utilizaba los palillos. Sin embargo, los bebés suecos, que no utilizan habitualmente los palillos, solo lograron anticipar el uso de la cuchara.

La evidencia disponible sugiere que el conocimiento acerca del uso de un objeto es importante para poder anticipar las acciones de los demás. Aunque estos fenómenos han sido magistralmente descritos por autores clásicos de la psicología del desarrollo (Baldwin, 1895/1906; Piaget, 1936/2009), no existen, según nuestro conocimiento, investigaciones que atiendan a las anticipaciones corporales que realizan los bebés en interacciones triádicas. El objetivo de este manuscrito es describir las anticipaciones corporales realizadas por bebés en las actividades triádicas conjuntas en las que participan comúnmente. El propósito último de este estudio es construir categorías de análisis que contribuyan con la realización de investigaciones longitudinales sobre el desarrollo de las anticipaciones corporales en contextos triádicos.

\section{Método}

\section{Participantes}

El presente artículo se basa en el análisis de una selección de videos recuperados de un estudio piloto más amplio que comprende un mayor número de observaciones longitudinales de los mismos niños/as. En dicho estudio participaron 4 niños/ niñas (niñas; $\mathrm{n}=3$ ) que fueron observados mensualmente desde los 6 hasta los 12 meses de edad. Las familias participantes pertenecían a la clase media Argentina. El máximo nivel educativo de los padres era "secundario completo" y su edad promedio de 34,5 años.

\section{Materiales y procedimiento}

Antes de la grabación, se obtuvo un consentimiento informado por escrito de los tutores de los bebés participantes. La selección de los sujetos del estudio fue intencional y se gestionó su participación a través de contactos personales de los investigadores.

Se observaron y filmaron distintas interacciones triádicas entre los bebés, uno de sus tutores y objetos de uso cotidiano en contexto ecológico (hogar) en la ciudad de Ensenada (Argentina). El marco metodológico empleado para la grabación 
audiovisual fue el de la observación no participante (Miller, 2017). La consigna dada a los tutores fue que dieran de comer a sus bebés tal como lo harían un día habitual, en un horario y en un sitio de la casa en que típicamente alimentaran a sus hijos/as. Cabe aclarar que no se dieron indicaciones adicionales ni se brindó ningún objeto. Aunque estas decisiones metodológicas dejan libradas ciertas variables (e.g., postura corporal de los participantes), su ventaja radica en que permiten preservar las condiciones ecológicas de la interacción, de modo que los participantes empleen sus propias estrategias interactivas y la actividad sea lo más natural posible. Por poner un ejemplo, la variable hambre del bebé no fue controlada en este estudio. No obstante ello, las observaciones fueron realizadas en horarios que los bebés eran alimentados habitualmente, según reportaron sus tutores. Esto permitió conservar las condiciones naturales de interacción y aumentó las probabilidades de que los niños tuvieran apetito al momento de la observación.

Para el registro de las observaciones uno de los investigadores acudía al hogar de las familias en el horario habitual en el que los bebés eran alimentados y filmaba empleando una cámara KODAK Modelo PlaySport. Cuando fue posible, la cámara se posicionó de forma tal que captara completamente al bebé, al adulto y a los distintos objetos empleados por ellos. Para la selección de las secuencias analizadas en este trabajo se consideraron los siguientes criterios: (i) que cuando el adulto llevase el objeto hasta la boca del niño/a su brazo/mano se acercase frontalmente y que fuera potencialmente visible para aquel; (ii) que la mirada del niño/a estuviese dirigida a la acción del adulto; (iii) que rostro y boca del niño/a fuesen claramente visibles; $y$, (iv) que el niño/a estuviese en condiciones de anticipar corporalmente el accionar del adulto (e.g. que no tuviese la boca llena de comida impidiendo que la abra o que estuviese atada/o a una silla que impidiera sus movimientos).

\section{Análisis de los datos}

Para la anotación y codificación de los comportamientos nos servimos del software ELAN (Versión 5.6, Lausberg y Sloetjes, 2009). Los comportamientos fueron codificados inicialmente a velocidad normal para identificar aspectos relevantes y luego cuadro a cuadro para identificar los puntos de inicio y finalización. El objetivo fue codificar la presencia/ausencia de anticipaciones corporales en el niño/niña antes de que la demostración de uso de objetos de los adultos finalizara. En otras palabras, se etiquetó un comportamiento como anticipación corporal cuando los bebés realizaron ajustes corporales complementarios con una acción instrumental del adulto incompleta (e.g., si el bebé abría anticipadamente su boca para recibir el biberón que le ofrecía su madre era considerado un caso de anticipación corporal). El inicio y fin de los episodios de las anticipaciones corporales fueron identificados como cambios comportamentales desde una posición normal en las siguientes regiones del cuerpo: torso: adelantamiento; brazos: extensión; cabeza: rotación del cuello o adelantamiento de la cabeza; boca: apertura. Para la codificación de los comportamientos anticipatorios se emplearon categorías que emergieron del 
proceso de análisis de los videos. Las categorías resultantes fueron las siguientes: (i) anticipaciones corporales tipo 1; (ii) anticipaciones corporales tipo 2; (iii) anticipaciones corporales tipo 3 (véase Tabla 1).

Tabla 1. Clasificación de anticipaciones corporales en interacciones triádicas

\begin{tabular}{lll}
\hline \multicolumn{1}{c}{ Categoría } & \multicolumn{1}{c}{ Definición } & \multicolumn{1}{c}{ Ejemplo } \\
\hline $\begin{array}{l}\text { Anticipaciones } \\
\text { corporales tipo 1 }\end{array}$ & $\begin{array}{l}\text { Configuraciones corporales que están } \\
\text { alineadas con el comportamiento futuro } \\
\text { del otro, pero no con el uso canónico del } \\
\text { objeto que es eje de la interacción. }\end{array}$ & $\begin{array}{l}\text { Adelantar el torso hacia la } \\
\text { cuchara con la que el adulto le } \\
\text { dará de comer. }\end{array}$ \\
$\begin{array}{l}\text { Anticipaciones } \\
\text { corporales tipo 2 }\end{array}$ & $\begin{array}{l}\text { Configuraciones corporales alineadas } \\
\text { con el comportamiento futuro del otro y } \\
\text { con el uso canónico del objeto que es eje } \\
\text { de la interacción. }\end{array}$ & $\begin{array}{l}\text { Abrir la boca antes de que el } \\
\text { adulto realice una demostración } \\
\text { de uso canónico con la } \\
\text { mamadera. }\end{array}$ \\
$\begin{array}{ll}\text { Anticipaciones } \\
\text { corporales tipo 3 }\end{array}$ & $\begin{array}{l}\text { que combinan elementos de las } \\
\text { anticipaciones específicas y otro registro } \\
\text { semiótico (e.g., vocalizaciones) }\end{array}$ & $\begin{array}{l}\text { "abrir la boca mientras se dice } \\
\text { realice una demostración de uso } \\
\text { canónico con la cuchara. }\end{array}$ \\
\hline
\end{tabular}

\section{Resultados}

Se seleccionaron 3 secuencias de video que resultan interesantes en el contexto de nuestro estudio. En ellas aparecen comportamientos que ejemplifican las categorías de análisis descritas anteriormente. Adicionalmente, se incluyó una secuencia en la que el niño/niña, a causa de su experiencia en el mundo, no anticipó corporalmente las acciones del adulto (Observación 1). Esta secuencia es importante no solo porque apoya la evidencia disponible, sino porque permite establecer distintas comparaciones con el resto de las observaciones. Todas las secuencias serán analizadas cualitativamente a continuación. La duración de las secuencias es de entre 4 s. y $21 \mathrm{~s}$.

\section{Observación 1: L. [0;6(8)] no realiza anticipaciones corporales antes de la demostración de uso del adulto (Tabla 2)}

Según reportaron los tutores de L., la interacción que observamos fue la primera ocasión en la que uno de ellos intentó alimentarlo empleando una cuchara ${ }^{1}$. Esto nos ofreció la oportunidad de observar la coordinación de comportamientos entre el adulto y el bebé alrededor de un objeto inédito en sus interacciones. Al no existir un historial de usos canónicos de la cuchara, resultaba esperable que el comportamiento de L. no estuviese alineado con las normas de uso de dicho objeto.

Inicialmente, el adulto intentó alimentar al bebé con la cuchara dos veces. Sin embargo, a pesar de que rozaba los labios del bebé con la cuchara, este no abrió su boca en ninguna de las dos oportunidades. Además, L. intentó tomar el objeto por su parte cóncava. Es decir que, en función de su experiencia en el mundo, L. respondió de forma no canónica a las demostraciones de uso de la cuchara de su 
padre. El comportamiento no canónico de L. resultaba totalmente esperable, dado que era la primera ocasión en que él recibía alimento semi-sólido con una cuchara. Sin embargo, su intervención interfería con la demostración de uso que intentaba realizar el adulto pues obstaculizaba su trayectoria. Acto seguido, el adulto evitó el intento de agarre del bebé y alejó la cuchara de su alcance.

Tabla 2. Observación 1. Duración: 21s.

Descripción de la acción
2. A. roza los labios de L. con la cuchara (intento de demonstración de
uso) y L. se muestra extrañado. El bebé intenta tomar la cuchara y A.
lo evita.
su parte cóncava (intento de demonstración de uso).

Posteriormente en la interacción el adulto introdujo la cuchara en la boca de L. sin que este anticipara corporalmente su accionar. Luego, L. exhibió desagrado (posiblemente por el sabor novedoso que estaba experimentando) y sacó la lengua expulsando de su boca la comida y el objeto. Finalmente, el adulto quitó el excedente de comida del rostro de L. con la cuchara.

En conclusión, observamos que L., un bebé que no estaba habituado al uso de las cucharas, no fue capaz de alinear su comportamiento con el del adulto cuando este intentó demostrar el uso de dicho artefacto. Por ejemplo, el bebé no anticipó corporalmente el accionar instrumental de su padre. Por sí sola la ausencia de anticipaciones no nos permite afirmar que el bebé no comprenda intencionalmente a los demás. El bebé podría comprender el sentido del comportamiento instrumental de su padre y no realizar anticipaciones corporales por otras razones. Sin embargo, la falta de anticipaciones se suma a una serie de comportamientos no canónicos de L. que apoyan la idea de que el niño no entendía las intenciones del adulto. En ese sentido, su comportamiento no resultó congruente con el uso canónico de la cuchara durante toda la secuencia. Por caso, cada vez que el adulto procuró alimentar al bebé con la cuchara, este último intentó tomar la cuchara por su parte cóncava, obstaculizando la demostración de uso del objeto.

Durante esta actividad triádica conjunta fue el adulto quien asumió la mayor parte de la responsabilidad. No solo organizó el escenario y la disposición corporal del 
bebé, sino que, además, realizó varias demostraciones incompletas de uso canónico (Alessandroni, 2020) seguidas por una demostración de uso completa de dicho objeto. Con "completa" hacemos alusión al hecho de que el adulto realizó todos los pasos que componen la demostración de uso de la cuchara: (i) cubrió toda la trayectoria que va desde el plato hasta la boca del bebé con la cuchara cargada; (ii) estimuló al bebé para que abriera su boca; (iii) introdujo la cuchara en la boca de L. sin que este la abriera; (iv) vació el contenido de la cuchara (inclinando la cuchara hacia arriba y haciendo pasar la superficie de esta sobre el labio superior del bebé); (v) limpió con la cuchara la comida que su hijo expulsó. Con estas intervenciones pedagógicas el adulto instala progresivamente un curso de acción culturalmente valorado (i.e., la cuchara se emplea para comer) y favorece la alineación del comportamiento del bebé con el mismo. Al mismo tiempo el adulto desalienta otros cursos de acción no canónicos (e.g., la cuchara con comida no se agarra por su parte cóncava) y que, si bien son esperables en niños/as de 6 meses de edad, resultan poco deseables a nivel cultural.

Observación 2: Am. [0;6(8)] realiza una anticipación corporal tipo 1 antes de la demostración de uso de la adulta (Tabla 3)

Tabla 3. Observación 2. Duración: 6s.

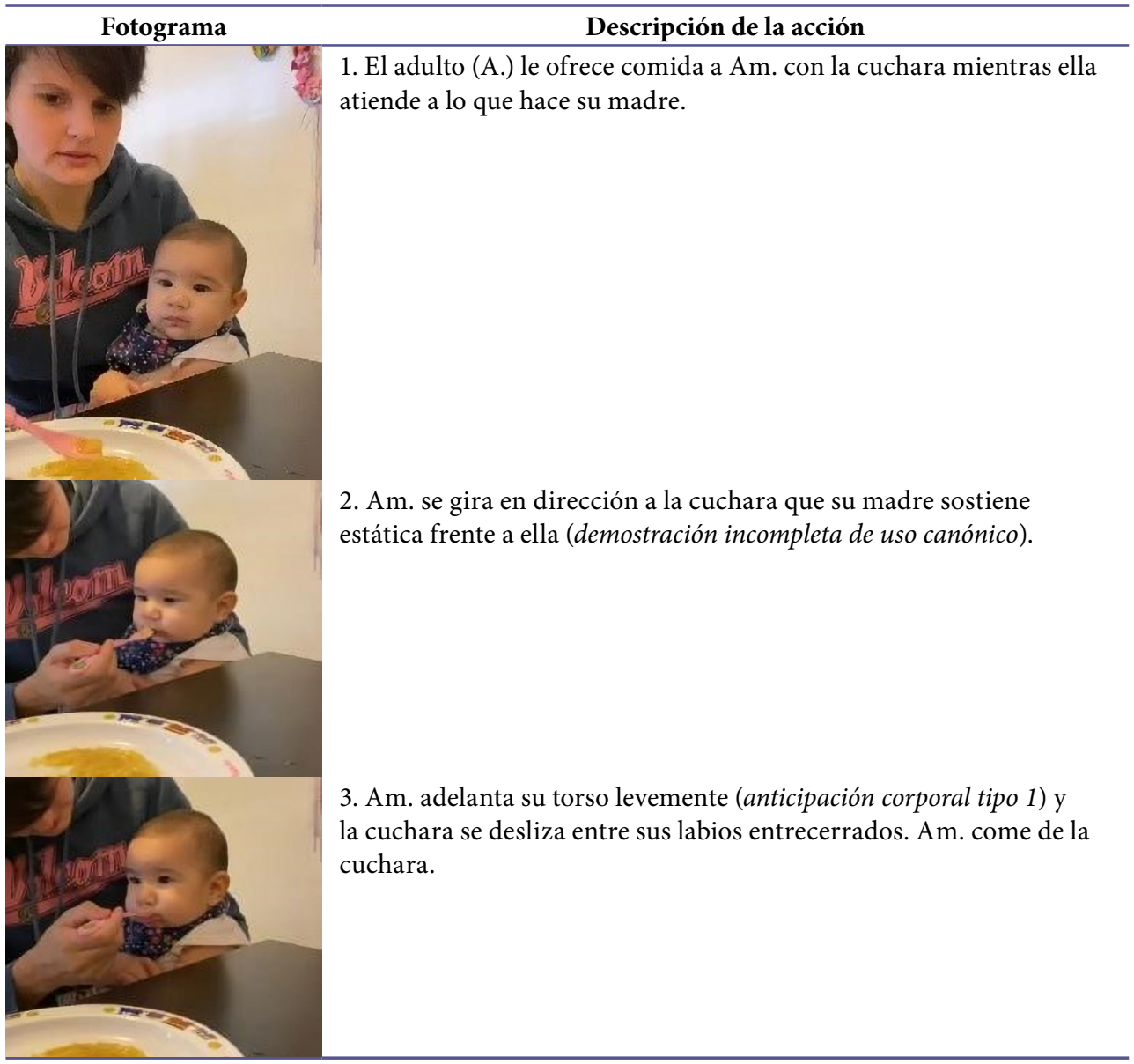


Cuando la adulta le ofreció de comer a Am., ella no se mantuvo indiferente ni obstaculizó el accionar de su madre, sino que respondió coherentemente, inclinando su torso hacia adelante e interceptando la trayectoria que la adulta realizaba con el objeto (anticipación corporal tipo 1).

Existen diferencias entre las Observaciones 1 y 2. En cuanto al comportamiento de los adultos, la madre de Am. realizó una demostración de uso de la cuchara menos completa. Por ejemplo, ella no inclinó la cuchara tan marcadamente hacia el final de la demostración de uso. Por el contrario, una vez que la cuchara estuvo dentro de la boca de la bebé, la sostuvo en posición horizontal por varios segundos, lo que posibilitó que fuera la bebé quien retirase el alimento de la cuchara (véase, Raczaszek-Leonardi et al., 2013). En cuanto al comportamiento de la bebé, ella exhibió una anticipación corporal tipo 1 antes de la que demostración de uso canónico de la cuchara finalizara. Esto involucra un mayor grado de autonomía y de apropiación cultural. La intervención de Am. da cuenta de la modificación paulatina y no reflexiva de los patrones de comportamiento de los bebés en relación a lo que hacen los demás y al uso de los objetos: se adelantó hacia la cuchara y acortó el recorrido que la adulta tuvo que realizar con el objeto. Una acción que inicialmente es completa responsabilidad del adulto, fue in-corporada en un comportamiento anticipatorio del bebé. De esta forma los bebés se introducen en las dinámicas de acción normativas vinculadas a un objeto, a pesar de no usarlo canónicamente por su cuenta.

En conclusión, esta secuencia puede ser vista como un episodio de comprensión intencional enactivo, corporeizado y mediado por la materialidad. Evidentemente, no estamos sugiriendo que la comprensión intencional haya tenido lugar a nivel intracraneal, sino en el involucramiento triádico mismo en el cuál adulto, bebé y objetos participan (Alessandroni, 2020, p. 17). Es decir que, cuando Am. y su madre interactuaron y se comunicaron con/sobre/a través (Alessandroni et al., 2019) de la cuchara lograron coordinar sus comportamientos: la adulta comenzó una demostración de uso canónico del objeto dirigida a la bebé y ésta última anticipó corporalmente el fin del accionar instrumental de su madre.

Observación 3: Ly. [0;6(9)] realiza anticipaciones corporales tipo 1 y 2 antes de una demostración de uso de la adulta (Tabla 4).

Aquí la adulta llamó la atención de la bebé sobre la cuchara y se la acercó. Acto seguido, Ly. abrió su boca ampliamente (anticipación corporal tipo 2) antes de que la cuchara tocara su boca. Este comportamiento constituye un caso de anticipación corporal de la bebé puesto que involucró la preparación del propio cuerpo para una acción de la adulta que aún no se había completado. Posteriormente, la adulta acercó más la cuchara hacia Ly. y la bebé inclinó su cuerpo hacia adelante (anticipación corporal tipo 1). Hacia el final de la secuencia, la adulta hizo una demostración de uso canónico inmediata de la cuchara, (i) introduciéndola en la boca de la bebé e (ii) inclinándola hacia arriba. Por último, Ly. cerró su boca alrededor de la cuchara. 
Tabla 4. Observación 3. Duración: 8 s.

Descripción de la acción
2. A. acerca la cuchara hacia Ly. y ella abre su boca (anticipación
cuchara. Ly. alza su mirada en dirección al objeto.
corporal tipo 2).

Al igual que en la Observación 1, advertimos aquí un caso de comprensión intencional mediado por la materialidad. No obstante, el comportamiento que exhibe la bebé en esta secuencia es más complejo y ajustado a las normas de uso canónico de la cuchara. Ly. realizó una anticipación corporal funcionalmente específica a la demostración de uso de la cuchara: abrió su boca. Además, al cerrar su boca hacia el final de la demostración de uso de la cuchara, la bebé evitó que la comida se cayera y contribuyó a lograr una interacción más fluida y coordinada.

\section{Observación 4: E. [0;11(12)] realiza una anticipación corporal tipo 3 antes de la demostración de uso de la adulta (Tabla 5)}

$\mathrm{Al}$ iniciar la secuencia E. señaló reiteradas veces hacia el pocillo de comida. Aunque podría haber realizado otros comportamientos como, por ejemplo, llorar o quejarse (los cuales también adoptó a lo largo de esa cena), articuló su deseo con gestos indiciales. Esto denota un grado creciente de apropiación de las normas culturales. Es decir, E. empleó un gesto que es aceptado culturalmente para solicitar algo que se desea. Este comportamiento es muy significativo en el contexto de nuestro trabajo por su relevancia a nivel interactivo y ontogenético. A nivel interactivo, el gesto indicial de E. orientó el comportamiento de la adulta y el devenir de la interacción en un sentido positivo. Es decir, con su intervención, E. hizo que un curso de acción específico sea sobresaliente para su madre (i.e., ofrecer comida con la cuchara) en detrimento de otras acciones posibles en ese escenario.

Tomando en consideración que el gesto indicial es cognitivamente más complejo que otros comportamientos comunicativos (e.g., reaching) y su emergencia es más tardía en el desarrollo (Moreno-Núñez et al., 2019), resta comprender la razón de su aparición en este contexto. El gesto indicial tiene la ventaja de orientar con mayor especificidad el accionar del otro, al reducir comparativamente más sus grados de 
libertad (i.e., haciendo que un curso de acción se destaque por sobre los demás). Cuando la bebé señaló el plato de comida durante la cena, la adulta no respondió de cualquier modo, aunque fuera coherente con el gesto de señalar (e.g., alcanzarle el pocillo y la cuchara a la bebé), sino que le ofrece comida específicamente. Es decir que, dado que E. logra hacerse comprender más fácilmente cuando usa gestos indiciales, al emplearlos economiza esfuerzos comunicativos y logra que los adultos satisfagan su deseo más rápidamente.

Tabla 5. Observación 4. Duración: 9s.

Descripción de la acción
indicial). Luego, señala el pocillo de comida con su otra mano (gesto
indicial);
2. El adulto (A.) dice "Ya, ya... ya te doy. Esperame un cachito".
E. lleva su pecho hacia adelante, levanta sus hombros y su mentón
(anticipación corporal tipo 1);

Continuando con la descripción de la interacción, en el momento en que la adulta se disponía a darle de comer E. dejó de señalar, adelantó su pecho, llevó sus brazos sutilmente hacia atrás e inclinó su cabeza (anticipaciones corporales tipo 1). La bebé detuvo sus demandas y esperó el arribo de la comida, mientras se preparaba corporalmente para una acción de la adulta que aún no había comenzado. Cuando la adulta terminó de dosificar la comida en la cuchara, la sopló y se la ofreció a E. Seguidamente, E. realizó más anticipaciones corporales: levantó más su mentón, inclinó su cabeza más hacia atrás y abrió su boca (alrededor de $3 \mathrm{~s}$. antes de que la cuchara tocara sus labios). Además, en el instante en que la cuchara estuvo frente a E., la bebé sopló la comida, tal como su madre le mostró que debía hacer (anticipación corporal tipo 3). La adulta, que observaba, asintió y dijo "Muy bien", confirmando o reforzando sus expectativas en relación con el comportamiento de E. Esto le permitiría a la bebé determinar con mayor precisión cuáles son los cursos de acción culturalmente privilegiados por su madre. Posteriormente, los comportamientos de la adulta y de la bebé alcanzaron un alto grado de sincronía: mientras E. abría su boca y adelantaba su cuerpo en dirección a la cuchara, la adulta adelantó su mano con la 
cuchara en dirección a la boca de la bebé. Finalmente, E. comió de la cuchara asistida por la adulta (demostración de uso canónico inmediata).

Es de interés remarcar ciertas diferencias de la interacción de E. con su madre con respecto a las secuencias precedentes. En primer lugar, las anticipaciones corporales que describimos anteriormente en otros sujetos comenzaban cuando las demostraciones de los adultos ya estaban en curso. Sin embargo, E. anticipó corporalmente la demostración de uso de la cuchara de su madre cuando este comportamiento no había siquiera comenzado. El aumento de la coordinación, marcado por anticipaciones corporales más largas que se adelantan al inicio del comportamiento del otro (y no solo a su finalización), podría ser considerado como un indicador de una mejor comprensión mutua. En segundo lugar, algunas de las anticipaciones corporales que exhibió E. a lo largo de la interacción dan cuenta de una mayor complejidad, pues involucran más partes de su cuerpo (e.g., la extensión hacia atrás de sus brazos) e incluyen nuevos comportamientos (i.e., soplar la comida antes de comerla). Esto daría cuenta de un mayor grado de normatividad y de ajuste con relación al comportamiento de los otros y del uso cultural de los objetos. En tercer lugar, E. realizó otros comportamientos no anticipatorios que pueden ser considerados como indicadores de comprensión intencional: gestos indiciales. Los gestos que realizó E. durante la interacción no solo nos muestran que ella podía tomar la iniciativa, sino que también podía reconocer lo que su madre era capaz de hacer con los objetos disponibles (i.e., cuchara y plato). En otras palabras, si E. señaló la cuchara para que su madre la alimente, es porque comprendía enactivamente que la adulta sabía cómo usar la cuchara. Concretamente, los gestos observados también constituyen un caso de atribución enactiva de conocimiento mediada por la materialidad.

\section{Discusión}

Estudios precedentes sugieren que las anticipaciones corporales que realizan los bebés durante su primer año de vida indican que ellos comprenden el comportamiento de los demás en términos intencionales (Fantasia et al., 2016; Reddy et al., 2013). Sin embargo, hasta la actualidad no se ha determinado si estos comportamientos también ocurren en las interacciones triádicas y cuáles serían sus características en caso de presentarse. En este estudio nos dimos el objetivo de describir cualitativamente las anticipaciones corporales que realizan los bebés en las interacciones triádicas tempranas. Definimos a las anticipaciones corporales como ajustes corporales complementarios de los bebés a una acción instrumental del adulto que aún no ha sido completada. Pusimos de manifiesto que, con base en su experiencia en el mundo, los bebés pueden anticipar corporalmente (e.g., adelantando el torso y/o abriendo su boca) las demostraciones de uso canónico de los objetos que los adultos dirigen intencionalmente hacia ellos.

Las dinámicas interactivas descritas en este trabajo, incluyendo, por caso, gestos y distintos niveles de complejidad de las anticipaciones corporales, parecen indicar 
que el conocimiento sobre el uso canónico de los objetos contribuye al desarrollo de la comprensión del comportamiento de los demás. Cuando los bebés saben para qué se usa un objeto pueden comprender más fácilmente lo que otros harán con dicho artefacto (véanse Alessandroni, 2020; Costall, 2013). Nuestros resultados apoyan la idea de que los objetos funcionan como signos materiales (Malafouris, 2013, 2019) que posibilitan formas complejas de comunicación y comprensión intersubjetiva. Los bebés no se representan mentalmente los estados intencionales de los adultos para luego ajustar la configuración de su cuerpo con relación al uso funcional de los objetos. Los ajustes corporales anticipatorios son per se una forma básica, corporeizada y enactiva de comprender a los demás. En otras palabras, proponemos que la comprensión intencional que desarrollan los bebés en sus primeros meses de vida ocurre a través de la coordinación de la propia acción con el comportamiento de los demás en concordancia con prácticas socio-materiales específicas (Alessandroni, en prensa; Vietri et al., enviado).

En nuestro trabajo diferenciamos, además, tres grados de complejidad de las anticipaciones corporales: anticipaciones corporales tipo 1, tipo 2 y tipo 3. En líneas generales, lo que distingue entre sí a estas modalidades es su complejidad, el grado de ajuste con relación al comportamiento del otro y del uso cultural del objeto. En otras palabras, cada una de estas presentaciones de las anticipaciones corporales resulta más elaborada que la anterior (e.g., a diferencia de las anticipaciones corporales tipo 1, las anticipaciones corporales tipo 2 involucran un ajuste con relación al uso canónico de los artefactos). Así como constatamos un componente dinámico en el comportamiento anticipatorio de los bebés, advertimos cambios en las intervenciones de los adultos. Ellos parecen descompletar sus demostraciones de uso de los objetos y favorecer un rol más activo por parte de los bebés. Por caso, si inicialmente introducían la cuchara dentro de la boca de los bebés, luego la dejan suspendida frente ellos/as para que éstos coman de allí (i.e., demostración incompleta de uso canónico) (Alessandroni, 2020).

Los comportamientos anticipatorios de los bebés ofrecen unas ventajas adaptativas que podrían favorecer su estabilización dentro del sistema interactivo. Por un lado, la emergencia de las anticipaciones corporales podría suponer una gran economía a nivel intersubjetivo (Rodríguez, 2006, p. 130), ya que, por caso, conllevan una reducción de los tiempos necesarios para realizar una demostración de uso. Además, las anticipaciones corporales adquieren significados normativos por su recurrencia en el tiempo (véase, Alessandroni, en prensa) y llegan a desempeñar un rol relevante en la comunicación adulto-bebé. Por ejemplo, para los adultos las anticipaciones corporales del bebé podrían funcionar como signos del involucramiento intersubjetivo del niño/a. Un bebé que anticipa la demostración de uso de una cuchara o de un biberón instancia su deseo de comer o beber. En ese caso, el comportamiento anticipatorio del bebé podría orientar el comportamiento del adulto (e.g., animándolo a ofrecer más comida/bebida) y mejorar la fluidez interactiva.

Por otro lado, a nivel intrasubjetivo, los bebés también podrían obtener una ganancia pragmática (Alessandroni y Rodríguez, 2019, p. 343) de las anticipaciones 
corporales. Por poner un ejemplo, si en lugar de permanecer inmóviles los bebés realizan anticipaciones corporales podrían lograr una satisfacción más rápida de sus necesidades alimenticias².

Una variable que podría haber impactado sobre las anticipaciones corporales es la postura de los participantes, junto con algunas de sus dimensiones como, por ejemplo, la ubicación física del bebé (i.e., sentado en una silla o sobre el regazo del adulto) o la posición del bebé respecto del adulto (i.e., sentado de frente a él o de espaldas) ${ }^{3}$. Quienes abogan por un enfoque corporeizado para el estudio del desarrollo humano (Marmeleira y Duarte-Santos, 2019), sostienen que el cuerpo y la acción tienen un rol constitutivo de la cognición humana (e.g., Thelen et al., 1987; Thelen y Smith, 1994/1996; Thelen y Spencer, 1998). Desde este punto de vista resulta coherente pensar que diversas posturas corporales ofrecen distintos grados de libertad y pueden determinar, a su vez, la emergencia de ciertos comportamientos en detrimento de otros (Thelen et al., 1987). En otras palabras, existirían condiciones interactivas que serían más favorables que otras para la emergencia de las anticipaciones corporales. Es dable suponer que las interacciones triádicas ocurridas frente a frente facilitarían episodios de involucramiento material entre adultos y bebés. Aunque estas condiciones no son estrictamente necesarias para la emergencia de anticipaciones corporales, la atención al rostro del compañero y la atención conjunta sobre un mismo objeto podrían ser factores que faciliten la emergencia de comportamientos anticipatorios en los niños/as.

Para finalizar es necesario decir que el estudio reportado en este manuscrito presenta una serie de limitaciones metodológicas (i.e., el tamaño de la muestra o el manejo de la temporalidad) que moderan el alcance sus resultados. Futuras indagaciones deberán corroborar la existencia de los comportamientos anticipatorios descritos aquí considerando, por ejemplo, la realización de observaciones longitudinales con muestras mayores, otras actividades y el uso de otro tipo de artefactos. También puede resultar de interés determinar si existe alguna conexión entre los tipos de anticipación corporal circunscritos en este trabajo, así como especificar posibles trayectorias de desarrollo de este comportamiento.

\section{Notas}

${ }^{1}$ Los padres de L. decidieron introducir la alimentación complementaria (i.e., alimentos semi-sólidos) a los 6 meses de edad de su hijo por recomendación de su pediatra de cabecera. Esta indicación del profesional es coherente con lo propuesto por la Sociedad Argentina de Pediatría respecto de la alimentación de los bebés durante el primer año de vida (Sociedad Argentina de Pediatría, 2001).

${ }^{2}$ En respuesta al señalamiento de una de las revisoras, aclaramos que no estamos negando la existencia de otros medios por los cuales los bebés lograrían que los adultos satisfagan sus necesidades (e.g., expresiones emocionales o gestos). En su lugar proponemos considerar, por un lado, que los bebés obtendrían beneficios concretos de las anticipaciones corporales y, por otro, que esas mismas ventajas podrían ser un factor relevante en la estabilización de dichos comportamientos a nivel interactivo.

${ }^{3}$ Agradecemos a ambas revisoras de Revista de Psicología UNLP por haber señalado este aspecto. 


\section{Referencias}

Abramova, E. y Slors, M. (2015). Social cognition in simple action coordination: A case for direct perception. Consciousness and Cognition, 36, 519-531. HTTPS://DOI.ORG/10.1016/. CONCOG.2015.04.013

Alessandroni, N. (2020). Object concepts and their functional core: Material engagement and canonical uses of objects in early childhood education. Human Arenas. HTTPS://DOI. ORG/10.1007/S42087-020-00119-5

Alessandroni, N. (en prensa). La semiotización cultural del cuerpo: Una base de la atribución enactiva de estados mentales. En N. Alessandroni y M. C. Piro (Eds.), Cuerpo, época $y$ presentaciones sintomáticas actuales. EDULP.

Alessandroni, N., Moreno-Núñez, A., Rodríguez, C. y Del Olmo, M. J. (2020). Musical dynamics in early triadic interactions: A case study. Psychological Research, 84(6), 1555-1571. HTTPS://DOI.ORG/10.1007/s00426-019-01168-4

Alessandroni, N. y Rodríguez, C. (2019). On perception as the basis for object concepts: A critical analysis. Pragmatics \& Cognition, 26(2-3), 321-356. HTTPS://DOI.ORG/10.1075/PC.19027.ALE

Baldwin, J. M. (1895/1906). Mental development in the child and the race. Methods and processes. Macmillan.

Costall, A. (2013). Things that help make us what we are. En G. Sammut, P. Daanen y F. M. Moghaddam (Eds.), Understanding the self and others (pp. 66-76). Routledge.

De Bruin, L. C., y Kästner, L. (2012). Dynamic embodied cognition. Phenomenology and the Cognitive Sciences, 11(4), 541-563. HTTPS://DOI. ORG/10.1007/S11097-011-9223-1

De Bruin, L., Strijbos, D., y Slors, M. (2011). Early social cognition: Alternatives to implicit mindreading. Review of Philosophy and Psychology, 2(3), 499-517. HTTPs://DOI. ORG/10.1007/S13164-011-0072-1

De Jaegher, H., Di Paolo, E., y Gallagher, S. (2010) Can social interaction constitute social cognition? Trends in Cognitive Sciences, 14(10), 441-447. HTTPS://DOI.ORG/10.1016/J.TICS.2010.06.009

ELAN (Version 5.6). (2019). Nijmegen: Max Planck Institute for Psycholinguistics. HTTPS://TLA.MPI NL/TOOLS/TLA-TOOLS/ELAN/.

Fantasia, V., Markova, G., Fasulo, A., Costall, A. y Reddy, V. (2016). Not just being lifted: Infants are sensitive to delay during a pick-up routine. Frontiers in Psychology, 6, 2065.
Gopnik, A. y Wellman, H. M. (1994/2002). La teoría de la teoría. En L. Hirschfeld, S. A. Gelman (Eds.) y A. Ruiz (Trad.), Cartografía de la mente: La especificidad de dominio en la cognición y en la cultura (pp. 3-63). Gedisa.

Green, D., Li, Q., Lockman, J. J. y Gredebäck, G. (2016). Culture influences action understanding in infancy: Prediction of actions performed with chopsticks and spoons in chinese and swedish infants. Child Development, 87(3), 736-746. HTTPS:/DOI.ORG/10.1111/CDEV.12500

Hobson, P. (2002). The cradle of thought: Exploring the origins of thinking. Macmillan.

Hunnius, S. y Bekkering, H. (2010). The early development of object knowledge: A study of infants' visual anticipations during action observation. Developmental Psychology, 46(2), 446-454. HTTPS://DOI.ORG/10.1037/A0016543

Hutto, D. D. (2011). Elementary mind minding, enactivist-style. En A. Seemann (Ed.), Joint attention: New developments in psychology, philosophy of mind, and social neuroscience (pp. 307-341). MIT Press. HTTPs://DOI.ORG/10.7551/ MITPRESS/8841.003.0016

Kochukhova, O. y Gredebäck, G. (2010). Preverbal infants anticipate that food will be brought to the mouth: An eye tracking study of manual feeding and flying spoons: Infants comprehension of feeding. Child Development, 81(6), 17291738. HTTPS://DOI.ORG/10.1111/J.1467$\underline{8624.2010 .01506 . \mathrm{X}}$

Lausberg, H. y Sloetjes, H. (2009). Coding gestural behavior with the NEUROGES-ELAN system. Behavior Research Methods, Instruments $y$ Computers, 41(3), 841-849. HTTPS ://DOI. ORG/10.3758/BRM.41.3.841

Lindblom, J. (2020). A radical reassessment of the body in social cognition. Frontiers in Psychology, 11, 987. HTTPS://DOI.ORG/10.3389/ FPSYG.2020.00987

Malafouris, L. (2013). How things shape the mind. A theory of material engagement. The MIT Press.

Malafouris, L. (2019). Mind and material engagement. Phenomenology and the Cognitive Sciences, 18(1), 1-17. HTTPS://DOI.ORG/10.1007/ s11097-018-9606-7

Miller, S. (2017). Developmental research methods (5th ed.). SAGE Publications.

Mireault, G. C. y Reddy, V. (2016). Humor in infants. Springer International Publishing. 
Moreno-Núñez, A., Rodríguez, C. y MirandaZapata, E. (2019). Getting away from the point: The emergence of ostensive gestures and their functions. Journal of Child Language, 1-23. HTTPS://DOI.ORG/10.1017/S0305000919000606

Newton, P., Reddy, V. y Bull, R. (2000). Children's everyday deception and performance on falsebelief tasks. British Journal of Developmental Psychology, 18(2), 297-317. HTTPS://DOI. ORG/10.1348/026151000165706

Onishi, K. H. y Baillargeon, R. (2005). Do 15-monthold infants understand false beliefs? Science, 308(5719), 255-258. HTTPS://DOI.ORG/10.1126/ SCIENCE.1107621

Piaget, J. (1936/2009). El nacimiento de la inteligencia en el niño (P. Bordonaba, Trad.). Crítica.

Raczaszek-Leonardi, J., Nomikou, I. y Rohlfing, K. J. (2013). Young children's dialogical actions: The beginnings of purposeful intersubjectivity. IEEE Transactions on Autonomous Mental Development, 5(3), 210-221. HTTPS://DOI. ORG/10.1109/TAMD.2013.2273258

Reddy, V. (1991). Playing with others' expectations: Teasing and mucking about in the first year. En A. Withen (Ed.), Natural theories of mind (pp. 143-158). Blackwell.

Reddy, V. (2001). Infant clowns: The interpersonal creation of humour in infancy. Enfance, 53(3), 247. HTTPS://DOI.ORG/10.3917/ENF.533.0247

Reddy, V. (2008). How infants know minds. Harvard University Press.

Reddy, V. y Mireault, G. (2015). Teasing and clowning in infancy. Current Biology, 25(1), R20-R23. HTTPS://DOI.ORG/10.1016/J.CUB.2014.09.021

Reddy, V., Markova, G. y Wallot, S. (2013). Anticipatory adjustments to being picked up in infancy. PLoS ONE, 8(6), e65289. HTTPS://DOI. ORG/10.1371/JOURNAL.PONE.0065289

Reddy, V., y Morris, P. (2004). Participants don't need theories: Knowing minds in engagement. Theory \& Psychology, 14(5), 647-665. HTTPs:// DOI.ORG/10.1177/0959354304046177

Rodríguez, C. (2006). Del ritmo al símbolo. Los signos en el nacimiento de la inteligencia. Horsori.

Rodríguez, C., Basilio, M., Cárdenas, K., Cavalcante, S., Moreno-Núñez, A., Palacios, P. y Yuste, N. (2018). Object pragmatics: Culture and communication - the bases for early cognitive development. En A. Rosa y J. Valsiner (Eds.), The Cambridge handbook of sociocultural psychology (2.a ed., pp. 223-244). Cambridge University Press.
Rossmanith, N. y Reddy, V. (2016). Structure and openness in the development of self in infancy. Journal of Consciousness Studies, 23(1-2), 237-257.

Rossmanith, N., Costall, A., Reichelt, A. F., López, B. y Reddy, V. (2014). Jointly structuring triadic spaces of meaning and action: Book sharing from 3 months on. Frontiers in Psychology, 5. HTTPS://DOI.ORG/10.3389/FPSYG.2014.01390

Schilbach, L., Timmermans, B., Reddy, V., Costall, A., Bente, G., Schlicht, T., y Vogeley, K. (2013). Toward a second-person neuroscience. Behavioral and Brain Sciences, 36(4), 393-414. HTTPS://DOI.ORG/10.1017/So140525X12000660

Sociedad Argentina de Pediatría (2001). Guía de alimentación para niños sanos de 0 a 2 años. Sociedad Argentina de Pediatría.

Spaulding, S. (2018). How we understand others: Philosophy and social cognition. Routledge.

Striano, T. y Reid, V. M. (2006). Social cognition in the first year. Trends in Cognitive Sciences, 10(10), 471-476. HTTPS://DOI.ORG/10.1016/J. TICS.2006.08.006

Surian, L., Caldi, S. y Sperber, D. (2007). Attribution of beliefs by 13-month-old infants. Psychological Science, 18(7), 580-586. HTTPS://DOI.ORG/10.1111/ $\mathrm{J} .1467-9280.2007 .01943 . \mathrm{X}$

Thelen, E., Kelso, J. A. S. y Fogel, A. (1987). Self-organizing systems and infant motor development. Developmental Review, 7(1), 39-65. HTTPS://DOI.ORG/10.1016/0273-2297(87)90004-9

Thelen, E. y Smith, L. (1994/1996). A dynamic systems approach to the development of cognition and action. MIT Press.

Thelen, E., y Spencer, J. P. (1998). Postural control during reaching in young infants: A dynamic systems approach. Neuroscience \& Biobehavioral Reviews, 22(4), 507-514. HTTPS://DOI. ORG/10.1016/s0149-7634(97)00037-7

Vietri, M., Alessandroni, N. y Piro, M. C. (2019). La perspectiva de segunda persona de la atribución de estados mentales: Una revisión sistemática de su estado actual de desarrollo. Psykhe, 28(2), 1-17. HTTPS://DOI.ORG/10.7764/PSYKHE.28.2.1280

Vietri, M., Alessandroni, N. y Piro, M. C. (enviado). Enactivismo y comprensión intencional: Una propuesta semiótica, corporeizada y material.

Wimmer, H. y Perner, J. (1983). Beliefs about beliefs: Representation and constraining function of wrong beliefs in young children's understanding of deception. Cognition, 13(1), 103-128. 